\title{
Michaelis-Gutmann Body
}

National Cancer Institute

\section{Source}

National Cancer Institute. Michaelis-Gutmann Body. NCI Thesaurus. Code C36016.

A round basophilic inclusion body that contains calcium and iron. It is found in the bladder wall of patients with malakoplakia. 